\title{
Histoedu, redes sociales e historia de la educación: el pasado pedagógico desde el presente educativo ${ }^{1}$
}

\section{Histoedu, social networks and history of education: pedagogical past from a present perspective}

\author{
Andrés Payà Rico 1, Josep M. Duart Montoliu 2, Santiago Mengual Andrés

\begin{abstract}
Dpto. de Educación Comparada e Historia de la Educación, Universitat de València, España. Andres.Paya@uv.es
2 Profesor de los Estudios de Psicología y Ciencias de la Educación, Universitat Oberta de Catalunya, España. jduart@uoc.edu
\end{abstract} \\ Dpto. de Educación Comparada e Historia de la Educación, Universitat de València, España. santiago.mengual@uv.es
}

\section{Resumen}

El presente artículo muestra algunas reflexiones y resultados iniciales a propósito de un proyecto de investigación I+D+i sobre el aprovechamiento de las TIC y las redes sociales para la investigación universitaria de la historia de la educación. Los avances logrados respecto a la mejora de la comunicación docente y científica, la significatividad del aprendizaje y la motivación entre la comunidad académica, nos llevan a defender la necesidad de consolidar lo que hemos venido a denominar la historia de la educación 2.0 propia de la sociedad digital. Las múltiples oportunidades para aprender y construir el conocimiento de manera compartida nos retan a generar conocimiento en red y a colaborar conjuntamente para: dar a conocer nuestras investigaciones, conocer otras formas de trabajar, compartir recursos y experiencias, intercambiar información y trabajar colaborativamente, etc. El convencimiento y la apuesta por este tipo de trabajo en red, nos han llevado a la creación de HistoEdu como un espacio de colaboración científica y pedagógica relativo a la historia de la educación como disciplina.

\section{Palabras Clave}

Historia de la Educación; Redes Sociales: Investigación; Comunicación Científica.

\begin{abstract}
This article presents some preliminary results and considerations about a R\&D project about the use of ICTs and social media to do research on educational history. The progress achieved regarding the improvement of social and scientific communication, the significance of the learning process and the motivation shown by the academic community, make us point out the need to consolidate what we have been referring to as history of education 2.0. There are many chances available to learn and to build up knowledge in a shared way, and they challenge us to generate network knowledge and to cooperate in order to: spread our research, get to know other approaches to work, share resources and experiences, exchange information, work collaboratively, etc. We believe in this type of virtual and shared way of working, which has lead us to create HistoEdu, a website to collaborate scientifically and pedagogically regarding the history of education as a discipline.
\end{abstract}

\section{Keywords}

History of Education; Social Network; Research, Scientific Comunication.

\footnotetext{
1 Este artículo se enmarca en el proyecto de investigación competitivo GV2013-089 "Patrimonio educativo 2.0: personal learning network and education heritage" subvencionado por la Conselleria d'Educació, Cultura i Esport del Gobierno Valenciano según Resolución de 28 de mayo de 2013 del director general de Universidades, Estudios Superiores y Ciencia, por la cual se conceden las ayudas para la realización de proyectos de I+D para grupos de investigación emergentes. IP: Andrés Payà (Universitat de València); Miembros del equipo de investigación: Santiago Mengual y Piedad M. ${ }^{a}$ Sahuquillo (Universitat de València), Pablo Álvarez (Universidad de Sevilla), Xavier Motilla (Universitat de les Illes Balears) e Isabel Gutiérrez (Universidad de Murcia) http://histoedu.es)
} 


\section{Introducción}

En los últimos años se han multiplicado considerablemente proyectos y experiencias que han intentado explorar y explotar las posibilidades que la Web 2.0 y las redes sociales ofrecen tanto para la investigación como para los procesos de enseñanza-aprendizaje en diversas áreas del conocimiento humano y en diversos ámbitos formativos, entre los cuales el universitario no ha sido una excepción (Alonso y Alonso, 2014; Cabero y Marín, 2014; García et al., 2013; Gewerc et al., 2014; Gonzalo et. al., 2012; Islas y Carranza, 2012). En el presente artículo damos cuenta de las implicaciones que todo ello ha tenido entre la comunidad científica de historiadores de la educación, repasando las principales aportaciones que se han desarrollado desde los inicios del siglo XXI, principalmente centradas en las posibilidades que ofrecen las TIC para la historia de la educación, así como para difundir entre un público amplio el patrimonio histórico-educativo. Las posibilidades que la Web 2.0 y las redes sociales ofrecen en la actualidad, a las cuales no ha sido ajena la comunidad científica de historiadores de la educación, planteaban la conveniencia de dar un paso más, partiendo de los hitos alcanzados principalmente en el último lustro, para llegar a lo que se ha venido a denominar como una historia de la educación 2.0 (Payà, 2012; Payà y Álvarez, 2013), en que investigadores, docentes y discentes compartan información, interactúen y creen conocimiento histórico-educativo.

\section{El uso de las TIC, internet y las redes sociales por los historiadores}

Desde hace algunas décadas, como sucede en el resto de ámbitos científicos, la historia no ha sido ajena a las posibilidades que las TIC ofrecen al historiador e investigador, podríamos citar, a modo de ejemplo del aprovechamiento inicial de la informática, el uso de sistemas automáticos de cálculo para tratar series voluminosas de datos numéricos y obtener indicadores estadísticos sobre la evolución cronológica de fenómenos históricos, de índole económica, demográfica, etc. De hecho, fue la escuela historiográfica francesa de los Annales quien asumió que era lícita la práctica de una historia cuantitativa y serial, que fue cobrando fuerza desde la década de 1960, apoyada en los cálculos obtenidos con ordenadores (Fernández Izquierdo, 2013).

Por otro lado, más allá de las posibilidades que la informática ofrecía a los historiadores desde mediados del siglo pasado para realizar una historia cuantitativa y serial, también han revolucionado, en el ámbito académico, la comunicación entre personas y la localización de información, documentación, referencias bibliográficas, etc. para investigadores. A principios de los años 80 del siglo pasado, se empezó a utilizar la mensajería electrónica o email entre los usuarios de universidades y centros de 
investigación. Se facilitaba, además, la consulta de bancos de datos, almacenados en ordenadores remotos denominados servidores, donde se ofrecían informaciones especializadas, o programas informáticos, cuyo acceso era público, o restringido a los usuarios autorizados, mediante protocolos de transferencia de ficheros. Aunque la verdadera revolución se produjo cuando Internet se abrió a un uso generalizado, tanto comercial como privado, superando el ámbito estrictamente universitario.

Esta primera etapa de Internet (Web 1.0) se caracterizaba por el predominio de puntos de consulta, servidores, etc. cuyos webmasters ofrecen a los usuarios o lectores informaciones en diferentes soportes digitales, de forma estática. El crecimiento de los servicios en la red ha sido exponencial desde entonces, facilitado por los progresos en las comunicaciones, que han aumentado considerablemente el flujo de datos, el desarrollo de aparatos informáticos cada vez más potentes y versátiles, y la generalización de protocolos de comunicación abiertos, lo que facilita la interconexión entre los recursos. Por ejemplo, si pensamos en recursos bibliográficos en la red, en el caso de catálogos de bibliotecas o hemerotecas, se ha podido pasar de suministrar solo el texto de las fichas descriptivas con la localización de la prensa periódica, libros, artículos, imágenes, etc., a ofrecer el contenido completo on line de cada vez más obras y bases de datos de publicaciones científicas o repertorios documentales en red. La edición electrónica ha tenido consecuencias en el ámbito académico en la divulgación de trabajos científicos a través de revistas indizadas en repertorios documentales nacionales e internacionales.

En este sentido, en los últimos años hemos asistido a la difusión de nuevas formas de comunicación en Internet (Web 2.0), en la que la información pasa a ser inmediata, descentralizada, interactiva, afectando a los medios tradicionales de difusión de los resultados de la investigación en general, y también de los historiadores en general y de los historiadores de la educación en particular. El paso de la red de redes jerárquica inicial y con páginas Web estáticas, que aún pervive en su diseño, a la Web 2.0, con la que convive, que ofrece mayores posibilidades de interactuación y colaboración entre usuarios, diseños centrados en ellos, compartición participativa de información, etc., y en consecuencia la proliferación de servicios asociados a ella como blogs, wikis, redes sociales, entornos para compartir recursos diversos, plataformas, educativas, etc. ha supuesto que una creciente cantidad de los contenidos sean generados y mantenidos de forma colectiva. Así pues, por ejemplo, muchas personas participan desinteresadamente en iniciativas colectivas de acceso abierto tan populares como la Wikipedia, que ha relegado en gran parte a las enciclopedias que existían en papel desde el siglo XVIII, o mantienen blogs de informaciones diversas, de entre las cuales también de interés para los historiadores en general y para los de la educación en particular, con gran éxito de visitas e interacciones de usuarios.

Diferentes instituciones y experiencias académicas con finalidades divulgativas, docentes, etc. hacen uso de redes sociales de uso generalizado como Facebook o Twitter, por ejemplo. Un número en aumento de docentes e investigadores del ámbito universitario, alrededor de trece millones, tienen 
perfiles en redes sociales específicamente académicas como ResearchGate o Academia, en donde principalmente comparten con el resto de la comunidad sus publicaciones, pero en las que también pueden crear debates o foros abiertos a la comunidad, en el caso de ambas o, incluso, en el caso de ResearchGate, compartir proyectos de trabajo en línea comunes dadas las posibilidades de la red social científica mencionada como herramienta profesional para el trabajo colaborativo con usuarios de prácticamente todas las disciplinas científicas y del conocimiento (Valladolid, 2010), entre ellas también de un número creciente de historiadores, mayoritariamente del mundo anglosajón.

En los últimos años han empezado a estudiarse también las posibilidades de las redes sociales, por ejemplo, el proyecto APTIC en España, que ha explorado las posibilidades de diseñar un red social para el colectivo sanitario, en código abierto y mediante el trabajo colaborativo entre organizaciones y usuarios individuales, si bien el grupo investigador impulsor ha acabado decantándose por abrir la red social específicamente diseñada a otras redes sociales o entornos para compartir recursos ya existentes como Facebook, Twitter y YouTube (Armayones, 2010; Hernández et al., 2014), en lo que parece ser una constante en los diversos proyectos llevados a cabo en este sentido, dada su mayor visibilidad, accesibilidad, participación e interacción por parte de los usuarios al ser herramientas conocidas y de uso generalizado. Además, un número creciente de investigaciones ha prestado atención al uso de las redes sociales a nivel institucional por parte de las universidades y a sus posibilidades para la docencia en dichas instituciones (Gewerc et al., 2014; Gonzalo et al., 2012; Islas y Carranza, 2012).

Centrándonos en las posibilidades de las redes sociales para la historia en general, recientemente se han publicado algunos análisis sobre las posibilidades de las redes sociales en la formación universitaria de los futuros historiadores, docentes e investigadores (Mitidieri, 2012), desde perspectivas diversas. En España, existen cada vez más experiencias docentes universitarias en el campo de la historia, general o sectorial, que integran o exploran las posibilidades de las redes sociales de uso generalizado entre los alumnos para hacer los procesos de enseñanza-aprendizaje más participativos y cooperativos, de entre los cuales podemos citar, a modo de ejemplo, las recientes experiencias del uso de Twitter en la docencia de historia económica en la Universidad de Oviedo (López Zápico y Tascón, 2013), o también en dicha universidad, de creación de un Personal Learning Enviroment (PLE) para la docencia en historia en la clase de Castellano para extranjeros, de fácil manejo para docentes y alumnos y en el que se integren las redes sociales y entornos de compartición de recursos de uso generalizado (Morán, 2013). Más allá de los ejemplos mencionados, veamos que incidencia han tenido las TIC entre la comunidad científica de historiadores de la educación en España, tanto para la docencia como investigación y difusión del patrimonio histórico-educativo, en los últimos años repasando brevemente las principales iniciativas llevadas a cabo. 


\section{El arte de la retórica}

En el caso del área de Historia de la Educación, a nivel internacional, recientemente han surgido interesantes investigaciones relacionadas con la historia en red y la escritura digital como el proyecto Erasums Life Long Learning Programme History on Line (HoL), en el que participaron universidades y centros de investigación de Italia, Alemania, España, Francia, Grecia, Hungría, Rumania y Turquía (Bandini y Bianchini, 2007), el estudio de los manuales escolares utilizando bases de datos y la Web 2.0 (Bianchini, 2014), el impacto de la tecnología en el estudio del patrimonio educativo (Thyssen y Priem, 2013), la creación de museos pedagógicos en línea (Álvarez y García, 2013), etc., pero, por lo que respecta a comunidades virtuales o redes de trabajo específicas, estas son escasas y, además, recurren generalmente a blogs, listas de discusión y otro tipo de herramientas similares. Es el caso de: HBO-Historische Bildungsforschung Online' (Bibliothek für Bildungsgeschichtliche Forshung of the Deutschen Instituts für Internationale Pädagogische Forschung y Sektion Historische Bildungsfurschung der DGfE, Alemania); H-Education² (History of Education Society, Estados Unidos); HENN-History of Education Network News ${ }^{3}$ (Katholieke Universiteit Leuven, Bélgica); H-Childhood ${ }^{4}$ (History of Children and Youth, Estados Unidos); History-Child-Family Archives ${ }^{5}$ (Gran Bretaña y Europa); y Child_Lit (Rutgers University of New Jersey, Estados Unidos). Se trata de experiencias implementadas en diferentes países y contextos y auspiciadas por instituciones relevantes en este ámbito, pero que no aprovechan plenamente las posibilidades de la Web 2.0 en cuanto a la comunicación e interacción que ofrecen las plataformas en línea basadas en software social, si bien recientemente desde la Universidad de Lovaina se ha empezado a plantear seriamente el papel que las humanidades digitales pueden desempeñar para la historia de la educación (Van Ruyskensvelde, 2014).

De otro lado, en el contexto español, tampoco existe una red social o espacio de colaboración científica específico con una plataforma tecnológica con las prestaciones suficientes para que la comunidad de historiadores de la educación de habla hispana puedan intercambiar información de calidad, recursos docentes, propuestas e intereses relacionados con la investigación, así como compartir proyectos, trabajar en red o dar una mayor visibilidad e impacto a sus publicaciones, lo cual consideramos que es necesario en la línea de lo que se ha venido a denominar Historia de la Educación 2.0 (Álvarez y Payà, 2013).

\footnotetext{
1 http://www.fachportal-paedagogik.de/hbo/ 
Sin embargo, sí que podemos señalar aquí algunos precedentes y proyectos previos, que sobre la historia de la educación en nuestro país y el aprovechamiento de las posibilidades que ofrece Internet se han llevado a cabo en nuestro pasado más reciente.

En España, nuestra comunidad científica ha ido progresivamente incorporando, tanto para la docencia como para la investigación, las TIC e Internet. Podemos encontrar diversos precedentes de compañeros que han incidido en las posibilidades que la sociedad de la información y el conocimiento ofrecían a la historia de la educación, que se han visto aumentadas en la actualidad exponencialmente gracias a la Web. Así, hace ya más de una década que se reflexionaba en la revista Educació i Història sobre las posibilidades de la denominada red de redes a propósito de los centros de documentación que en ella se pueden encontrar (Miró, 2003), así como la reflexión sobre el impacto que historia de la educación iba a recibir con el uso generalizado de las TIC (Lázaro, 2003). Por entonces, también desde la UNED se vinculaban ya Internet e historia de la educación con los museos pedagógicos y el patrimonio educativo, a través de un estudio sobre la presencia de los museos escolares en Internet como exposiciones de patrimonio educativo (Somoza y Ossenbach, 2003). Fruto del creciente interés que en los últimos años suscitaron las posibilidades de Internet entre la comunidad científica de historiadores de la educación en España, surgieron los primeros estudios al respecto, como la investigación llevada a cabo por el Museo Pedagógico de Galicia sobre los museos de la educación en Internet (Peña, 2004). De manera paralela, comenzaron a organizarse exposiciones virtuales escolares, (Rabazas, 2006) y a remarcar la eclosión constante de los sitios webs relacionados con la historia de la educación (Dávila et al., 2005). Asimismo, para fomentar que historiadores de la educación y el público en general pudiesen acceder a información y documentación de bases de datos histórico-educativos en línea, desde la Universidad del País Vasco se puso en marcha un recurso para la historia de la educación en Euskadi on line, un proyecto que con el tiempo fue perfeccionándose hasta convertirse en el Centro de Documentación de Historia de la Educación en Euskal Herria (Dávila y Naya, 2009).

Poco a poco, el desarrollo de la tecnología educativa fue consolidándose en el discurso históricoeducativo, como puede apreciarse en el monográfico de la revista interuniversitaria «Historia de la Educación» sobre nuevas tendencias en historia de la educación, donde se constataba el desarrollo y la implantación de las TIC en nuestro ámbito académico y científico y la consecuente transformación a que sometía, y sometería, a la museología educativa (Ruiz Berrio, 2006). Sin embargo, las experiencias antes mencionadas se centraron, en mayor medida, en los aspectos investigadores y de recuperación del patrimonio educativo, y no tanto en la difusión de la investigación histórico-educativa. Otros trabajos desarrollados en los últimos años han incidido en elementos como el trabajo docente, la participación comunitaria o su utilización para la enseñanza-aprendizaje (Álvarez, 2009, 2011, Payà, 2010, 2011), no siendo hasta hace muy poco que se ha comenzado a insistir en la importancia de internet y las redes sociales para la comunicación científica y el trabajo en red (Payà y Álvarez, 2012, 2013, 2014). 


\section{HistoEdu.es: una red social histórico-educativa}

Una vez analizado el estado de la cuestión sobre la vinculación de las TIC y las redes sociales con la historia de la educación, exponemos a continuación una iniciativa investigadora y científica sobre la materia que nos ocupa, fundamentada y ejemplificada en la segunda fase del proyecto I+D+i "Patrimonio Educativo 2.0: Personal Learning Network of Education Heritage" (GV/2013/089 Generalitat Valenciana). En la fase inicial de dicho proyecto se delimitaron las necesidades de la construcción de una red social específica para los docentes-investigadores del área de Historia de la Educación. En este sentido se realizó un estudio descriptivo (Mengual et al., 2015) que recogió las impresiones de 58 profesores e investigadores universitarios pertenecientes a diversas asociaciones científicas directamente vinculadas con la Historia de la Educación (Sociedad Española de Historia de la Educación -SEDHE-, Sociedad Española para el Estudio del Patrimonio Histórico-Educativo -SEPHEy Societat d'Història de l'Educació dels Països de Llengua Catalana -Institut d'Estudis Catalans). La muestra del estudio inicial comprendió un total de 26 instituciones de educación superior del contexto español, así como un total de 5 instituciones extranjeras. El estudio se desarrolló con el instrumento Heritage-KNOWv1, construido y validado para tal efecto.

El objetivo esencial de la primera fase inicial fue detectar los conocimientos de los participantes sobre las redes sociales específicas de investigación, así como determinar los elementos que esta debía contener. El instrumento se construyó sobre ocho grandes áreas o dimensiones: a) Conocimiento/ uso redes sociales; b) Espacios web para la creación/difusión de contenido; c) Importancia de las TIC en los procesos de enseñanza-aprendizaje en HE; d) Factores en la elección de recursos TIC para la docencia en HE; e) Importancia de los tipos de medios audiovisuales para la docencia en HE; f) Posibilidades que ofrecen las TIC para el desarrollo del profesional en HE; g) Utilidad de herramientas en una red social académica/investigadora; h) Afinidad de uso de la red social.

Los hallazgos principales de la primera fase (Mengual et al., 2015), consistente en la detección de necesidades y expectativas, permitieron obtener la información suficiente para determinar las necesidades para la construcción de la plataforma/red social de investigación "HistoEdu.es", adaptándola a las funcionalidades, actitudes y preferencias de la comunidad científica y docente de historiadores de la educación.

\subsection{Elección de la plataforma para el desarrollo de HistoEdu.es}

Tras la fase inicial se abordó la segunda fase, basada inicialmente en una revisión teórica y práctica de las plataformas/herramientas OpenAcces que permitían desplegar una red social con una 
infraestructura asequible. En el proceso de selección de la plataforma se desecharon las herramientas SAS (Simple as Service) dado que el objeto era la personalización de una herramienta y no el consumo estandarizado de una aplicación.

Son muchas las herramientas y plataformas que actualmente permiten desplegar una red social genérica o específica bajo el paraguas del OpenAcces. De entre las herramientas más populares encontramos Elgg, Pligg, Buddypress (plugin para Wordpress), GNUSocial, JamRoom, etc. En dicho sentido tal vez Elgg sea la que más acogida haya tenido en los últimos años en los proyectos, programas e implementaciones académicas (e.g. : Coll, Engel, Sanz \& Bustos, 2014; Baillie et al., 2011; Lu, Churchil, 2013; Montes, Gea, Bravo-Lupiáñez, Molina, Bergaz \& Ramos, 2013), aunque BuddyPress no deja de ser una alternativa interesante, modular y flexible, tal y como algunos proyectos han puesto de manifiesto (e.g. : Paraskevas \& Stergatu, 2012; Santos, Brogueira, \& Bernardino, 2014; Stern \& Willits, 2011). Sin embargo, tras un periodo de evaluación, valoración y testeo se optó por emplear el framework CMS genérico Joomla dada su versatilidad a la hora de adaptarlo e implementarlo como red social, tal y como ha descrito otros proyectos recientes con objetivos similares (Huang, Zhang, Feng, Ju, Wei, Song, \& Xu, 2014; Priefer, 2014).

Durante el periodo de testeo de Joomla y sus variantes (forks), tras un análisis bibliométrico se detecto la existencia de una variante académica de Joomla empleada en numerosos casos de éxito para la implementación de redes sociales académicas o de investigación. En dicho sentido se optó por la

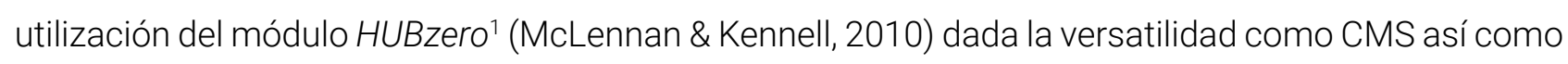
sus posibilidades como red social y, específicamente, por la alta disponibilidad de módulos enfocados a la construcción de una red social con herramientas para el docente-investigador. Entre los ejemplos más famosos de investigación y aplicaciones desarrolladas con este fork contamos con "nanoHUB" (Madhavan, Zentner \& Klimeck, 2013; Klimeck, McLennan, Brophy, Adams, \& Lundstrom, 2008), sin dejar de lado otros proyectos e investigaciones generados a partir de este fork específico (e.g. : Kalyanam, Zhao, Song, Wong, Lee \& Villoria, 2013; Kuriyan \& Reklaitis, 2013; Merwade, Feng, Zhao \& Song, 2012; Nanda, Tan, Auyeung \& Lehto, 2012; Nanda, Tan, Auyeung \& Lehto, 2013; Witt \& Yu, 2012; Zentner et al. 2011; Zhang, Maron \& Charles, 2013)

\subsection{Herramientas de HistoEdu.es}

Son diversas las posibilidades y módulos que HUBzero ofrece al implementarse con Joomla (McLennan \& Kennell, 2010). Tras la fase de implementación se decidió incorporar aquellas funcionalidad básicas de la red social y, tras un periodo de testeo (Beta), añadir otros módulos que fueran de utilidad para la comunidad histórico-educativa que iba a interactuar en esta red social. En esencia el portal “HistoEdu. 
es" permite al usuario registrado:

- Acceder al alta de novedades/eventos públicos/privados que podrá gestionar y que aparecerán en la web de forma automática.

- Repositorio de contenido público: emplear la plataforma como repositorio de contenido abierto (OER), pudiendo enlazar o publicar material académico o investigador en las categorías de: a) cursos; b) presentaciones; c) publicaciones, d) seminarios, e) otros materiales. Del mismo modo los administradores pueden confeccionar cursos completos al estilo MOOC enlazando los materiales existentes en la sección del repositorio.

- Generación de wikis: los usuarios registrados podrán crear y modificar documentos "wiki", gestionando el control sobre el mismo, así como los historiales.

- Búsquedas por TAGS: posibilita al usuario buscar cualquier contenido de la red social a través de etiquetado mediante palabras clave.

- Módulo de preguntas y respuestas (base de conocimientos). Permite a los usuarios establecer un sistema de comunicación, generación y respuesta de dudas y/o consultas.

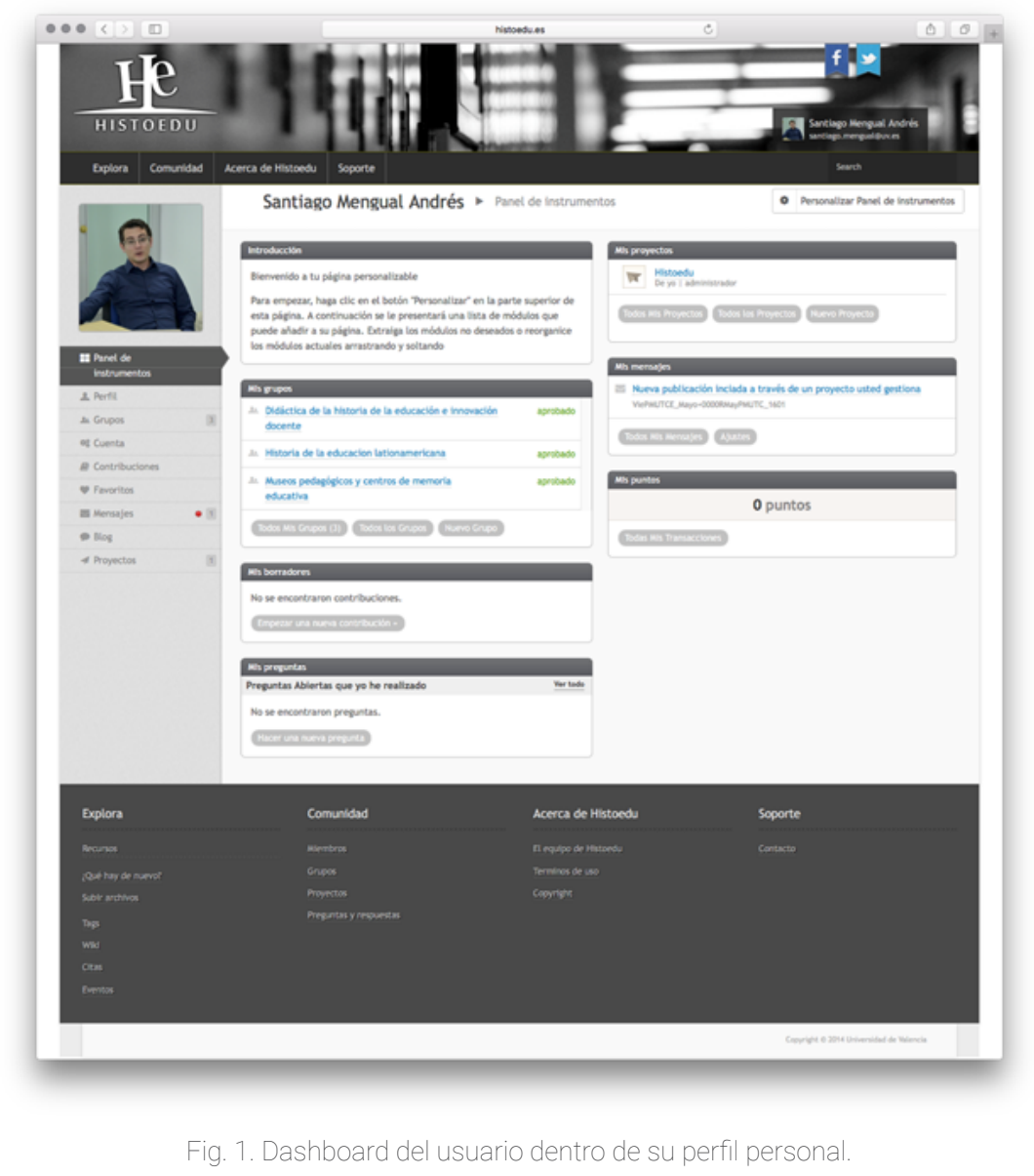


- Posibilidad de crear un perfil académico/investigador que dará opciones para (ver Figura 1): a) acceder a un panel de instrumentos que visualiza la actividad del usuario en el sitio; b) configurar la biografía del autor, c) crear grupos de trabajo público/privado; e) gestionar o subir las contribuciones personales al repositorio de documentos; f) gestionar los elementos marcados como "favoritos" dentro de la red social; g) sistema de mensajería/correo internos para la comunicación con los miembros de la red; e) blog personal del autor con la posibilidad de personalizar la visibilidad de las entradas (públicas/privadas); f) acceso a la creación de proyectos.

- Módulo grupos (ver Figura 2): este módulo permite la creación de grupos públicos/privados con un alto grado de personalización. El propietario podrá determinar quién puede unirse al grupo de trabajo (abierto a cualquiera o público, restringido, solo invitación o cerrado). Al mismo tiempo existirá la opción de configurar la visibilidad del grupo (visible/oculto), lo que determinará su presencia o no dentro de la red social general. Del mismo modo, los grupos permiten a su administrador configurar qué módulos estarán disponibles dentro del grupo y quién tendrá acceso a los mismos. Entre las herramientas que los grupos pueden disponer encontramos: a) directorio de miembros, b) wiki, c) recursos, d) debates, e) blogs, f) lista de deseos, g) proyectos, h) anuncios. A nivel particular cabe destacar que la opción proyectos permitirá las mismas opciones que la herramienta "proyectos" del perfil individual, con la particularidad de que los proyectos de los grupos están enfocados al trabajo en grupo.

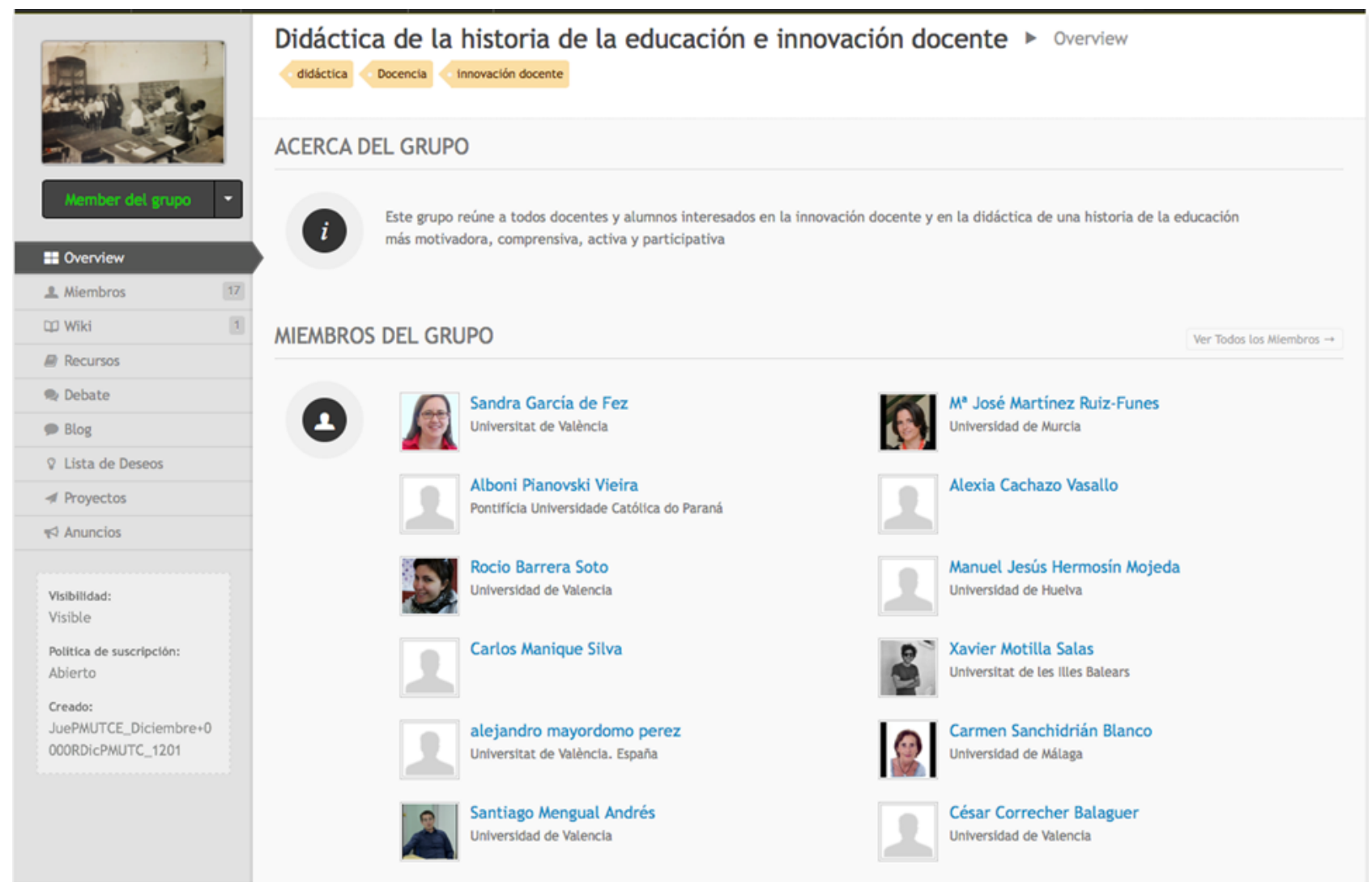


- Módulo proyectos (ver Figura 3): este módulo posee características comunes que se comparten para la gestión de proyectos personales. En esencia permite crear un espacio para la gestión de proyectos de cualquier tipo, bien sea de tipo individual o compartido con otros miembros de la comunidad, o bien la creación de proyectos dentro de algún grupo de trabajo ya existente. El propietario del grupo tendrá la posibilidad de añadir nuevos miembros (administradores o colaboradores). Desde la pantalla inicial de proyectos se obtendrá una visualización de la información o actividad reciente al estilo red social. De entre las herramientas del módulo proyectos encontramos: la sección de repositorio de archivos de trabajo, herramienta de asignación de tareas, sección para crear notas/subnotas empleando formato de trabajo wiki y, por último, la carga de publicaciones, working papers o documentos de trabajo del proyecto que serán depositados en el repositorio público.

\begin{tabular}{l}
\hline Histoedu (histoedu) \\
\hline Actualizaciones \\
\hline Información \\
\hline Archivos
\end{tabular}

\section{Publicaciones}

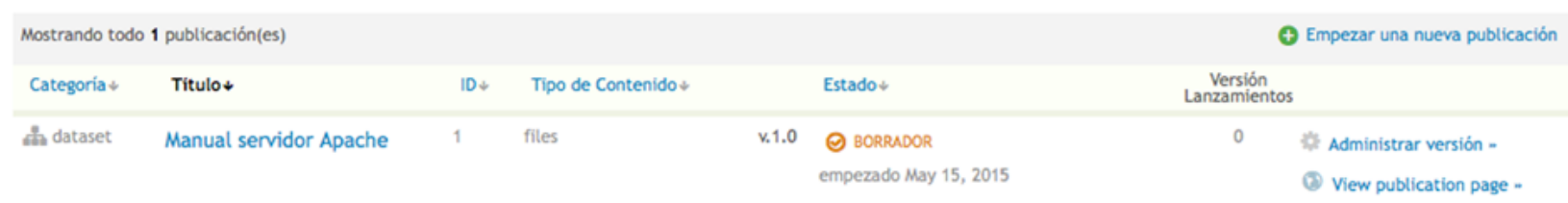
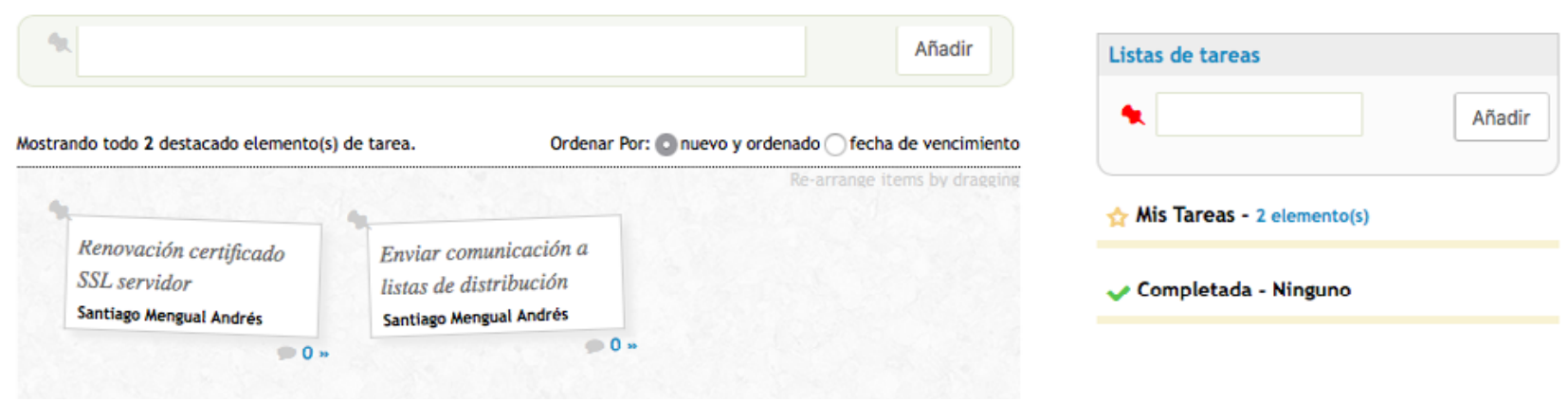

Fig. 3. Dashboard módulo proyectos, sección tareas y publicaciones. 


\section{Algunas conclusiones y desafíos de futuro}

Histoedu está pensada como una comunidad libre, construida con software libre, que pretende dar cabida a docentes, investigadores y personas interesadas en la Historia de la Educación. Supone en este sentido un desafío y compromiso público por ofrecer un espacio de conexión académico/ investigador que pretende llegar a ser sostenible con fondos públicos y contribuir a la difusión y transferencia del conocimiento científico histórico-educativo.

En la actualidad el proyecto se encuentra en su tercera fase. En la fase de pruebas (Beta) se están localizando posibles errores técnicos y/o lagunas de contenido de la plataforma e implementando progresivamente novedades que mejoren y completen la versión actual. De entre las novedades a incorporar en un futuro más inmediato, encontraremos la posibilidad de login mediante proveedores de terceros (Google Accounts, Facebook Account, etc). La fase de pruebas está resultando esencial y muy gratificante, pues estamos aprendiendo y mejorando desde la interacción del usuario final. En dicho sentido, la plataforma cuenta en la actualidad con más de 100 usuarios (todos ellos vinculados a Historia de la Educación), una aceptación que consideramos muy positiva atendiendo al número de profesionales tanto españoles como iberoamericanos y, en menor medida, de otros países europeos, interesados en dicha temática.

Muchos son los retos y mejoras que todavía quedan por implementar. De entre las dificultades encontradas hasta la fecha, cabe destacar la labor que se ha realizado contribuyendo a la traducción del fork. La versión de HUBZero original no disponía de una versión en lengua castellana, por lo que se ha destinado un tiempo considerable del proyecto a proveer una traducción del 80\% sobre la totalidad de sus módulos. Limitaciones de tipo presupuestario, así como problemáticas de otra índole, han impedido la completa adaptación lingüística, por lo que en la actualidad se está finalizando paulatinamente la traducción al mismo tiempo que estamos corrigiendo los errores, propuestas y sugerencias que reportan los usuarios en interacción con los administradores.

Histoedu es, sin lugar a dudas, un espacio abierto de aprendizaje compartido que responde a las necesidades detectadas en la fase inicial del proyecto (Mengual, et al., 2015) y que se postula como una herramienta que pretende mejorar el trabajo cooperativo y las relaciones académicas intra e internacionales, la visibilidad e impacto de las investigaciones histórico-educativas de sus miembros, así como la optimización de los procesos de gestión y comunicación científica (Duart \& MengualAndrés, 2014). 


\section{Referencias}

Alonso, S., \& Alonso, M. del M. (2014). Las redes sociales en las universidades españolas. Vivat Academia, 126, 54-62. doi: http://dx.doi.org/10.15178/va.2014.126.54-62

Álvarez, C., \& García, M. (2013). Un museo pedagógico en internet: diseño, desarrollo y evaluación. TESI. Teoría de la Educación: Educación y Cultura en la Sociedad de la Información, 14(2), 192-227.

Álvarez, P. (2009). El Museo Didáctico Virtual del Patrimonio Histórico-Educativo Andaluz como catedral de la memoria histórico-educativa de Andalucía. Foro de Educación. Pensamiento, cultura y educación, 11, 275-286.

Álvarez, P. (2011). Museos Virtuales de Pedagogía, Enseñanza y Educación: hacia una didáctica del patrimonio histórico-educativo. EARI. Educación Artística. Revista de Investigación, 2, 23-27.

Álvarez, P., \& Payà, A. (2013). Patrimonio educativo 2.0: hacia una didáctica histórico-educativa más participativa y la investigación en red. Cuestiones Pedagógicas, 22, 117-140.

Armayones, M., Hernández, E., Gómez, B., Guillamon, N., Ontiveros, G., Bosque, A., \& Nafría, B. (2010). APTIC: a social network to improve the quality of life of members of patients associations. Orphanet Journal of Rare Diseases, 5(1). doi: http://dx.doi.org/186/1750-1172-5-S1-P27

Baillie, S., Kinnison, T., Forrest, N., Dale, V. H. M., Ehlers, J. P., Koch, M., . . Van Beukelen, P. (2011). Developing an online professional network for veterinary education: The NOVICE project. Journal of Veterinary Medical Education, 38(4), 395-403. doi:http://dx.doi.org/10.3138/jvme.38.4.395

Bandini, G., \& Bianchini, P. (2007). Fare storia in rete. Fonti e modelli di scrittura digitale per la storia dell'educazione, la storia moderna e la storia contemporánea. Roma: Carocci.

Bianchini, P. (2014). The databases of school textbooks and the Web 2.0. History of Education and Children's Literature - HECL, 9 (1), 125-134. doi:http://dx.doi.org/10.1400/221588

Cabero, J., \& Marín, V. (2014). Posibilidades educativas de las redes sociales y el trabajo en grupo: Percepciones de los alumnos universitarios. Comunicar: Revista científica iberoamericana de comunicación y educación, 42, 165-172. doi:http://dx.doi.org/10.3916/C42-2014-16

Coll, C., Engel, A., Saz, A., \& Bustos, A. (2014). Personal learning environments: Design and use. Cultura y Educación, 26(4), 775-801. doi:http://dx.doi.org/10.1080/11356405.2014.985935 
Dávila, P., \& Naya, L. M. (2009). El Centro de Documentación de Historia de la Educación en Euskal Herria en Internet: una experiencia innovadora. Cuadernos de Historia de la Educación, 6, 111-123.

Dávila, P., Garmendia, J., Naya, L. M., \& Zabaleta, I. (2005). Un recurso de Internet para la historia de la educación en Euskal Herria: euskalhezkuntza.info. En P. Dávila \& L.M. Naya (Eds.), La infancia en la historia: espacios y representaciones (II), (pp. 555-563). San Sebastián: Erein y SEDHE.

Duart, J. M. \& Mengual-Andrés, S. (2014). Impact of the Knowledge Society in the University and in Scientific Communication. RELIEVE, 20(2), art. M4. doi:http://dx.doi.org/10.7203/relieve.20.2.4343

Fernández Izquierdo, F. (2013). Archivos, bibliotecas, redes sociales, blogs, Twitter... Tecnologías de la información al servicio del historiador modernista en la Web 2.0. En E. Serrano Martín (Ed.), De la tierra al cielo. Líneas recientes de investigación en Historia Moderna, (pp. 109-158). Zaragoza: Institución Fernando el Católico, CSIC y Diputación de Zaragoza.

García, C., Navarrete, M.C., \& Ancona, M.C. (2013). Las comunidades de aprendizaje y redes sociales en las universidades.Etic@net, 13-14,86-93.

Gewerc, A., Montero, L., \& Lama, M. (2014). Colaboración y redes sociales en la enseñanza universitaria. Comunicar: Revista científica iberoamericana de comunicación y educación, 42, 55-63. doi:http://dx.doi. org/10.3916/C42-2014-05

Gonzalo, J., Laser, W., \& Adrián, E. (2012). El uso de redes sociales por parte de las universidades a nivel institucional. Un estudio comparativo, Revista de Educación a Distancia, 32(11), 1-38.

Hernández, E., Gómez, B., Guillamon, N., Boixadós, M., \& Armayones, M. (2014). Analysis of Patients organizations needs and ICT use -The APTIC project in Spain to develop an online collaborative social network. Health Expectations, 1-14. doi:http://dx.doi.org/10.1111/hex.12181

Huang, L., Zhang, J., Feng, K., Ju, P., Wei, Y. F., Song, D. K., \& Xu, H. (2014). Building a scientific social network with joomla!: Vol. 687-691. Applied Mechanics and Materials (pp. 2316-2319).

Islas, C., \& Carranza, M. R. (2012). Uso de las redes sociales como estrategias de aprendizaje ¿Transformación educativa? Apertura. Revista de Innovación Educativa, 3(2), Recuperado el 21 de abril de 2014 de http://www.udgvirtual.udg.mx/apertura/index.php/apertura3/article/view/198/213

Kalyanam, R., Zhao, L., Song, C. X., Wong, Y. L., Lee, J., \& Villoria, N. B. (2013). Idata: A community geospatial data sharing environment to support data-driven science. Paper presented at the ACM International Conference Proceeding Series. doi:http://dx.doi.org/10.1145/2484762.2484813

Klimeck, G., McLennan, M., Brophy, S. P., Adams III, G. B., \& Lundstrom, M. S. (2008). nanoHUB.org: 
Advancing Education and Research in Nanotechnology. Computing in Science \& Engineering, 10(5), 1723. doi:http://dx.doi.org/10.1109/MCSE.2008.120

Kuriyan, K., \& Reklaitis, G. V. (2013). Creating shared resources for pharmaceutical technology education-Simulation tools. Education for Chemical Engineers, 8(4), e124-e131. doi:http://dx.doi. org/10.1016/j.ece.2013.10.002

Lázaro, L. M. (2003) L'accés a les fonts a la història de l'educació a través de les noves tecnologies de la informació. Educació i Història. Revista d'història de l'educació, 6, 245-264.

López Zápico, M.A., \& Tascón, J. (2013). El uso de Twitter como herramienta para la enseñanza universitaria en el ámbito de las ciencias sociales. Un estudio de caso desde la historia económica, TESI. Teoría de la Educación. Educación y Cultura en la Sociedad de la Información, 14(2), 316-345.

Lu, J., \& Churchill, D. (2013). Creating personal learning environments to enhance learning engagement. Paper presented at the Proceedings of the 2013 IEEE 63rd Annual Conference International Council for Education Media, ICEM 2013. doi:http://dx.doi.org/10.1109/cicem.2013.6820194

Lu, J., \& Churchill, D. (2014). The effect of social interaction on learning engagement in a social networking environment. Interactive Learning Environments, 22(4), 401-417. doi:http://dx.doi.org/10.10 80/10494820.2012.680966

Madhavan, K., Zentner, M., \& Klimeck, G. (2013). Learning and research in the cloud. Nat Nano, 8(11), 786-789. doi:http://dx.doi.org/10.1038/nnano.2013.231

McLennan, M., \& Kennell, R. (2010). HUBzero: A Platform for Dissemination and Collaboration in Computational Science and Engineering. Computing in Science \& Engineering, 12(2), 48-53. doi:http:// dx.doi.org/10.1109/MCSE.2010.41

Mengual-Andrés, S., Payá, A., \& Roig, R. (2015). Evaluación de necesidades y expectativas ante la construcción de un espacio interactivo de comunicación, información y aprendizaje históricoeducativo. Revista Complutense de Educación, 26 (Núm. Especial), 141-158. doi:http://dx.doi. org/10.5209/rev_RCED.2015.v26.46331

Merwade, V., Feng, W., Zhao, L., \& Song, C. X. (2012). WaterHUB: a resource for students and educators forlearning hydrology Proceedingsof the 1 st Conference of the ExtremeScienceand Engineering Discovery Environment: Bridging from the eXtreme to the campus and beyond. New York, USA: ACM. doi:http:// dx.doi.org/10.1145/2335755.2335857

Miró, I. (2003). Les noves tecnologies al servei de l'ensenyament de la història de l'educació. Educació i Història. Revista d'història de l'educació, 6, 240-244. 
Mitidieri, G. (2012). TIC en el aprendizaje de la historia: utilización e historización de estas herramientas en el capitalismo cognitivo. Clío \& Asociados, 16, 195-208.

Montes, R., Gea, M., Bravo-Lupiáñez, D., Molina, S., Bergaz, R., \& Ramos, A. (2013). Turning out a social community into an e-Learning platform for MOOC: The case of AbiertaUGR. Paper presented at the ACM International Conference Proceeding Series. doi:http://dx.doi.org/10.1145/2536536.2536611

Morán, P.M. (2013). La enseñanza de historia en el aula de ELE: construcción de un PLE adaptado al profesorado poco experto. Historia y Comunicación Social, 18, 587-599.

Nanda, G., Tan, J., Auyeung, P., \& Lehto, M. (2012). Evaluating HUBzero ${ }^{\text {TM }}$ as a collaboration platform for reliability engineering. Paper presented at the 62nd IIE Annual Conference and Expo 2012.

Nanda, G., Tan, J., Auyeung, P., \& Lehto, M. (2013). Improving efficiency of organizational reliability engineering knowledge using keywords. Paper presented at the IIE Annual Conference and Expo 2013.

Paraskevas, M., \& Stergatu, H. (2012). Design and implementation of a successful social networking educational platform. Paper presented at the Proceedings of the IADIS International Conference e-Learning 2012.

Payà, A. (2010). El patrimonio valenciano en la red. Un espacio virtual de aprendizaje para la historia de la educación. En E. Collelldemont (Ed.), Memoria, ciudadanía y museos de educación, (pp. 131-141). Vic: Universitat de Vic, MUVIP y SEPHE.

Payà, A. (2011). El patrimoni historicoeducatiu i la plataforma digital patrimonieducatiuvalencia.com. Un projecte de tots. En A. Mayordomo, M. C. Agulló y G. García (Ed.), El patrimoni historicoeducatiu valencià, (pp. 267-274). Valencia: CEIC Alfons el Vell y Universitat de València.

Payà, A. (2012) Historia de la educación 2.0: las TIC al servicio de la docencia y el aprendizaje en la Educación Superior. En J. M. Hernández Díaz (Ed.), Formación de élites y educación superior en Iberoamérica (s. XVI-XXI), (pp. 695-702). Salamanca: Hergar Ediciones, Antema.

Payà, A., \& Álvarez, P. (2012). Pensar la educación desde las TIC y la recuperación del patrimonio educativo. En O. Fontal (Ed.), Mirando a Europa: estado de la cuestión y perspectivas de futuro, (pp. 546554). Madrid: Ministerio de Educación, Cultura y Deporte.

Payà, A. y Álvarez, P. (2013). Historia y patrimonio de la educación 2.0: conocimiento compartido, recursos y propuestas didácticas. En G. Espigado et al. (Eds.), La Constitución de Cádiz. Genealogía y desarrollo del sistema educativo liberal, (pp. 799-810). Cádiz: Universidad de Cádiz y SEDHE.

Payà, A., \& Álvarez, P. (2014). Ús professional i possibilitats docents de les xarxes socials a la història 
de l'educació. «HistoEdu»: un espai de col·laboració científica. En F. Comas, S. González, X. Motilla, S. Sureda (Eds.), Imatges de l'escola, imatge de l'educació, (pp. 459-468). Palma de Mallorca: Universitat de les Illes Balears.

Peña, V. (Ed.) (2004). Os museos da educación en Internet. Santiago de Compostela: Xunta de Galicia y MUPEGA.

Priefer, D. (2014). Model-driven development of content management systems based on Joomla Proceedings of the 29th ACM/IEEE international conference on Automated software engineering (pp. 911 914). New York, USA: ACM. doi:http://dx.doi.org/10.1145/2642937.2653474

Rabazas, T. (2006). El Quijote en la escuela. Exposición virtual de MANES. Boletín informativo SEPHE, 1, 34-36.

Ruiz Berrio, J. (2006). Historia y museología de la educación. Despegue y reconversión de los museos pedagógicos. Historia de la Educación. Revista Interuniversitaria, 25, 271-290

Santos, C., Brogueira, G., \& Bernardino, C. (2014). Social networks with BuddyPress Proceedings of the International Conference on Information Systems and Design of Communication (pp. 182-183). New York, USA: ACM. doi:http://dx.doi.org/10.1145/2618168.2618202

Somoza, M., \& Ossenbach, G. (2003). Internet y museos pedagógicos, En R. Calvo et al. (Eds.), Etnohistoria de la escuela, (pp. 901-914). Burgos: Universidad de Burgos y SEDHE.

Stern, D. M., \& Willits, M. D. D. (2011). Social media killed the Ims: Re-imagining the traditional learning management system in the age of blogs and online social networks. Vol. 1. Cutting-Edge Technologies in Higher Education (pp. 347-373).

Thyssen, G., \& Priem, K. (2013). Mobilising meaning: multimodality, translocation, technology and heritage. Paedagogica Historica: International Journal of the History of Education, 49 (6), 735-744. doi:http://dx.doi.org/10.1080/00309230.2013.848912

Valladolid, I. (2010). ResearchGATE, un Facebook para científicos, Naukas, Consultado el 18 de abril de 2014 de http://naukas.com/2010/11/12/researchgate-un-facebook-para-cientificos

Van Ruyskensvelde, S. (2014). Towards a history of e-ducation? Exploring the possibilities of digital humanities for the history of education. Paedagogica Historica: International Journal of the History of Education, 50(6), 861-870. doi:http://dx.doi.org/10.1080/00309230.2014.955511

Witt, M., \& Yu, Y. (2012). Refactoring HUBzero for linked data. Paper presented at the Proceedings of the ACM/IEEE Joint Conference on Digital Libraries. doi:http://dx.doi.org/10.1145/2232817.2232845 
Zentner, L. K., Clark, S. M., Smith, P. M., Shivarajapura, S., Farnsworth, V., Madhavan, K. P. C., \& Klimeck, G. (2011). Practical Considerations in Cloud Utilization for the Science Gateway nanoHUB. org Proceedings of the 2011 Fourth IEEE International Conference on Utility and Cloud Computing (UCC). Victoria, NSW, 5-8 Dec. 2011 (pp. 287-292). USA: IEEE. doi:http://dx.doi.org/10.1109/UCC.2011.46

Zhang, T., Maron, D. J., \& Charles, C. C. (2013). Usability Evaluation of a Research Repository and Collaboration Web Site. Journal of Web Librarianship, 7(1), 58-82. doi:http://dx.doi. org/10.1080/19322909.2013.73904 